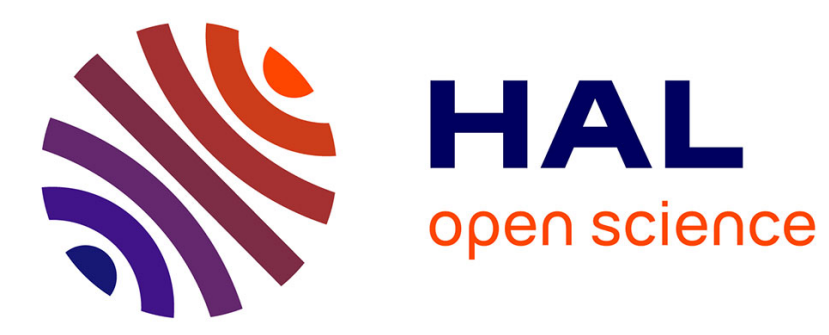

\title{
Simulation EHD des précipitateurs électrostatiques
}

\author{
A.C. Lahjomri, P. Atten
}

\section{To cite this version:}

A.C. Lahjomri, P. Atten. Simulation EHD des précipitateurs électrostatiques. Revue de Physique Appliquée, 1987, 22 (9), pp.1087-1094. 10.1051/rphysap:019870022090108700 . jpa-00245648

\section{HAL Id: jpa-00245648 https://hal.science/jpa-00245648}

Submitted on 1 Jan 1987

HAL is a multi-disciplinary open access archive for the deposit and dissemination of scientific research documents, whether they are published or not. The documents may come from teaching and research institutions in France or abroad, or from public or private research centers.
L'archive ouverte pluridisciplinaire HAL, est destinée au dépôt et à la diffusion de documents scientifiques de niveau recherche, publiés ou non, émanant des établissements d'enseignement et de recherche français ou étrangers, des laboratoires publics ou privés. 
Classificâtion

Physics Abstracts

$47.65-52.80-86.70$

\title{
Simulation EHD des précipitateurs électrostatiques
}

\author{
A. C. Lahjomri et P. Atten \\ Laboratoire d'Electrostatique et de Matériaux Diélectriques (*), \\ Centre National de la Recherche Scientifique, avenue des Martyrs, 166 X, 38042 Grenoble Cedex, France
}

(Reçu le 22 décembre 1986, accepté le 5 mars 1987)

\begin{abstract}
Résumé. - On examine le fonctionnement aérodynamique d'un précipitateur électrostatique compte tenu des connaissances actuelles sur les différents régimes d'électroconvection. On montre que la turbulence dans un précipitateur est essentiellement d'origine électrohydrodynamique et que l'intéraction entre les particules chargées de petite taille et le mouvement turbulent du gaz est tout à fait analogue à celle existant entre les ions injectés et la turbulence de la plupart des liquides diélectriques. Une simulation expérimentale, avec un liquide, d'un précipitateur à deux étages est définie. Une étude expérimentale est présentée et ses résultats sont comparés à des prédictions théoriques récentes.
\end{abstract}

\begin{abstract}
The aerodynamic functioning of an electrostatic precipitator is examined in the light of present knowledge on the different regimes of electroconvection. It is shown that the turbulence occurring in a precipitator basically arises from electrohydrodynamic processes and that the interaction between charged particles of small size and the turbulent flow of the gas is quite similar to the interaction between injected ions and turbulence in dieletric liquids. An experimental simulation, using a liquid, of a two stages precipitator is then defined. An experimental study is presented and its results are compared to recent theoretical predictions.
\end{abstract}

\section{Introduction.}

Certains des processus fondamentaux intervenant dans le fonctionnement des précipitateurs électrostatiques (PES) : charge des particules, constitution de couches plus ou moins conductrices, etc... sont bien identifiés et relativement bien compris. Par contre les phénomènes aérodynamiques intervenant dans la zone de collection des particules n'ont pas reçu une description satisfaisante. Ceci est dû en particulier à la méconnaissance des interactions complexes entre l'écoulement gazeux, les ions et les particules chargées soumises à l'influence du champ électrique appliqué. Ces interactions, qui sont de nature électrohydrodynamique (EHD) sont généralement sousestimées ou même ignorées. Les seules études théoriques faites jusqu'à présent ont supposé que l'effet de l'écoulement turbulent pouvait être décrit en terme de diffusion turbulente $D_{\mathrm{t}}$ supposée infinie (théorie de Deutsch [1]) ou finie (théorie de Léonard et al. [2]).

Dans les PES industriels l'écoulement gazeux est

(*) Laboratoire associé à l'Univ. Scient. Tech. et Méd. de Grenoble. turbulent. Dans la plupart des travaux antérieurs cette turbulence est attribuée à l'effet de l'écoulement forcé. L'idée principale de ce travail est de tenir compte de l'intensité de la force qu'exerce le champ sur la charge d'espace (créée par les ions et les particules chargées) et de la turbulence qui en résulte. De plus cette turbulence va réagir de manière très différente sur les répartitions des ions gazeux et des particules chargées. Nous allons voir que la turbulence d'origine électrique est généralement prépondérante et rend bien compte des observations expérimentales. Par ailleurs l'interaction entre les particules chargées et le mouvement turbulent de l'air est tout à fait analogue à l'interaction entre les ions et la turbulence de la plupart des liquides diélectriques. On montre à la suite d'un examen quantitatif que les particules chargées dans l'air se comportent comme les ions dans les liquides.

2. Modèles du fonctionnement aérodynamiques des PES.

La théorie de Deutsch [1] est habituellement utilisée pour estimer l'efficacité des précipitateurs et pour déterminer les caractéristiques des nouveaux PES 
construits. Cette théorie suppose que l'écoulement du gaz est turbulent avec une vitesse moyenne uniforme $U_{0}$, et que la densité de particules est uniforme dans la direction transversale (ceci revient à admettre que la diffusivité turbulente est infinie). La concentration de particules décroît comme $\exp \left(-x w_{\mathrm{E}} / U_{0} d\right)$, où $w_{\mathrm{E}}=K \bar{E}$ est la vitesse de migration des particules ( $d$ : distance entre électrodes, $K$ : mobilité des particules, $\bar{E}$ : champ électrique moyen). Dans la pratique la longueur $L$ caractérisant la décroissance exponentielle de la concentration de particules est souvent plus grande que la longueur de Deutsch $L_{\mathrm{D}}=U_{0} d / w_{\mathrm{E}}$.

D'importants travaux ont été réalisés dans le but de mieux tenir compte des phénomènes aérodynamiques intervenant dans la précipitation et ensuite d'améliorer la théorie de Deutsch. Léonard et al. [2] ont analysé la collection des particules en utilisant le concept de diffusivité turbulente pour rendre compte de l'effet de la turbulence sur le transport des particules. Ils ont montré que l'efficacité du PES doit dépendre d'un nombre de Peclet électrique turbulent $P e=K \bar{E} d / D_{\mathrm{t}}$ qui mesure l'importance respective de la migration des particules et de leur entraînement dû à la turbulence (Fig. 1). La théorie de Deutsch correspondant à $P e=0$ donne une borne inférieure de l'efficacité. On note que pour

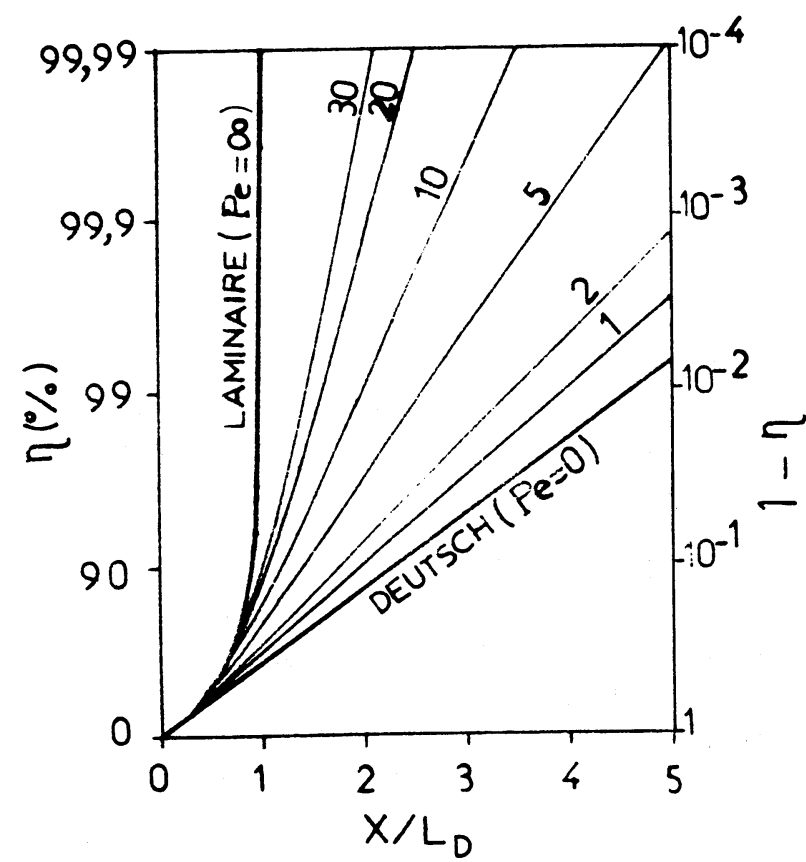

Fig. 1. - Variation théorique de l'efficacité en fonction du nombre de Deutsch $\left(x / L_{\mathrm{D}}=w_{\mathrm{E}} x / \bar{U} d\right)$ pour différentes valeurs du nombre de Peclet correspondant à un champ de collection uniforme.

[Theoretical efficiency curves as a function of Deutsch number $\left(x / L_{\mathrm{D}}=w_{\mathrm{E}} x / \bar{U} d\right)$ for different values of the Peclet number for a uniform-field collector.] une diffusivité finie on a un rendement meilleur que celui prédit par Deutsch.

Le problème qu'il reste alors à résoudre est de déterminer la diffusivité turbulente $D_{\mathrm{t}}$. Self et al. [3] ont admis que la turbulence résulte uniquement de l'écoulement forcé et ont proposé de prendre pour $D_{t}$ l'expression semi-empirique bien connue en mécanique des fluides. Plusieurs expériences $[4,5]$ ont paru appuyer cette idée d'une turbulence engendrée par l'écoulement principal. Il faut remarquer cependant que ces expériences ont été réalisées dans des conditions où la charge d'espace est due seulement aux ions avec très peu de particules. Dans ces conditions on a simplement superposition d'un écoulement forcé et du vent ionique. A la différence de Self et al. nous pensons que la turbulence est essentiellement d'origine électrohydrodynamique (EHD) et qu'en outre les particules chargées jouent un rôle important dans sa génération. Pour appuyer cette affirmation, nous allons rappeler brièvement les faits principaux en EHD dans les liquides diélectriques, et essayer de tirer des conclusions sur la génération de la turbulence dans les PES et les effets de cette turbulence sur le transport des porteurs de charge [6].

\section{Rappels sur l'électroconvection dans les liquides diélectriques en injection unipolaire.}

Sous certaines conditions l'action du champ électrique sur une couche plane de fluide diélectrique entraîne un mouvement intense du fluide [7]. Le problème de stabilité EHD consiste à examiner les interactions entre perturbations de densité de charge et de vitesse $[8,9]$. Le mouvement se déclenche audessus d'un seuil $T_{\mathrm{c}}$ du nombre adimensionnel $T=\varepsilon V / K \eta(\varepsilon:$ constante diélectrique ; $V:$ tension appliquée ; $\eta$ : viscosité). $T_{\mathrm{c}}$ décroît quand l'intensité de l'injection augmente. Pour un liquide donné, $T$ dépend seulement de la tension appliquée. Dans le cas d'une injection très forte (limitée par charge d'espace) dans l'air, la tension critique est de l'ordre de $40 \mathrm{kV}$ lorsque les porteurs de charge sont des ions et de quelques dizaines à quelques centaines de volts lorsque ce sont des particules chargées. Le point déterminant pour évaluer l'influence de la convection sur le transport de charge est de comparer l'amplitude des fluctuations de vitesse $w^{\prime}$ à la vitesse propre des porteurs de charge $K \bar{E}[10]$. Ceci amène à introduire la mobilité adimensionnelle $M=$ $(\varepsilon / \rho)^{1 / 2} / K$ ( $\rho$ : masse volumique du fluide). Dans le cas d'une injection très forte on trouve que $w^{\prime}=K_{\text {ehd }} \bar{E}$ avec $K_{\text {ehd }} \approx 1 / 3(\varepsilon / \rho)^{1 / 2}$. Pour $M<3$ (cas des ions dans l'air), les trajectoires des porteurs de charge sont très légèrement perturbées par le mouvement du fluide et le courant qui passe n'est pratiquement pas modifié. Par contre pour $M>3$ (cas des ions dans les liquides), les porteurs de 
charge peuvent être entraînés par le mouvement du fluide contre l'action du champ électrique. Dans ce cas la distribution de la charge moyenne est bouleversée et le transport convectif des porteurs de charge accroît fortement le courant moyen qui traverse la couche fluide [10].

Dans les géométries à champ divergent telles que lame-plan [11] et fil entre deux plaques [12, 13], il existe un mouvement pour toute valeur de $T(>0)$. La vitesse moyenne du jet de liquide pour une forte injection est de l'ordre de $K_{\text {ehd }} \bar{E}$. Dans les liquides l'écoulement est très turbulent et l'intensité turbulente peut atteindre 25 à $35 \%$. L'effet d'entraînement des porteurs de charges dépend toujours du nombre $M$ (Fig. 2).

(a)
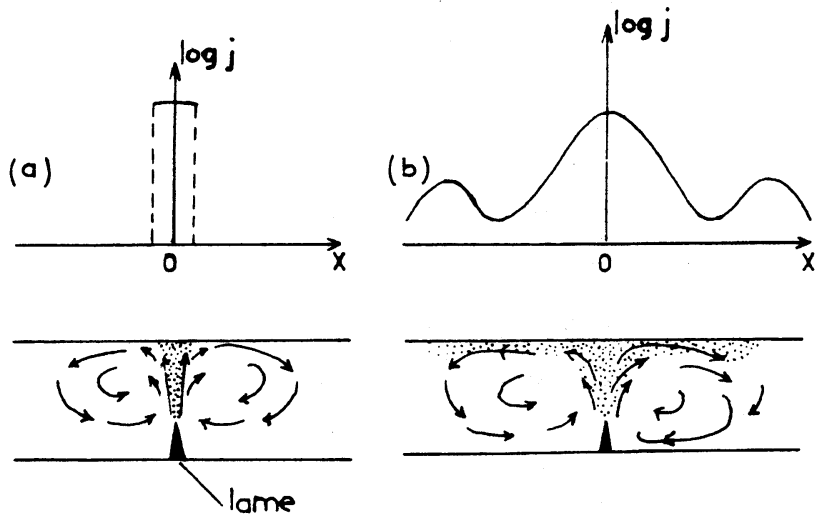

Fig. 2. - Injection par la lame en géométrie lame-plan : représentation schématique des lignes de courant fluide induites et de la distribution de densité de courant électrique sur l'électrode plane. a) Vent ionique dans un gaz $(M<3)$.b) Injection d'ions dans un liquide isolant $(M>3)$.

[Injection by the blade in a blade-plate geometry : schematic of induced stream-lines and distribution of electrical current density on the plane electrode. a) Ionic wind in a gas $(M<3)$. b) Injection of ions in an insulating liquid $(M>3)$.]

\section{Estimation des valeurs de $M$ pour des particules chargées dans l'air.}

Les phénomènes électroaérodynamiques (EAD) et EHD apparaissent en général différents. Cependant la distinction cruciale est celle relative à la valeur du paramètre $M$. Pour pouvoir caractériser le régime d'agitation, il faut d'abord déterminer les valeurs des mobilités des particules chargées dans un gaz (air) et les valeurs de $M$ correspondantes.

Considérons des particules sphériques. Leur vitesse $v_{\mathrm{p}}$ sous l'influence d'un champ électrique $E$ est obtenue en égalant la force de Coulomb $Q_{\mathrm{p}} E$ à la force de frottement dans l'air $F_{\mathrm{f}}$ :

$$
Q_{\mathrm{p}} E=\pi a^{2} \rho v_{\mathrm{p}}^{2} f / 2
$$

et la mobilité est :

$$
K_{\mathrm{p}}=v_{\mathrm{p}} / E=\left(Q_{\mathrm{p}} / \pi a^{2}(1 / 2) \rho f E\right)^{1 / 2}
$$

$f$ est le facteur de friction, $a$ le rayon de la particule, et $Q_{\mathrm{p}}$ sa charge limite. Nous utilisons la loi de Stokes quand le nombre de Reynolds formé avec $v_{\mathrm{p}}$ est inférieur à 1 :

$$
Q_{\mathrm{p}} E=6 \pi \eta a v_{\mathrm{p}} \text { et } K_{\mathrm{p}}=v_{\mathrm{p}} / E=Q_{\mathrm{p}} / 6 \pi \eta a
$$

$\eta$ et $\rho$ étant respectivement la viscosité dynamique et la masse volumique de l'air. Pour des particules de diamètre inférieur à $1 \mu \mathrm{m}$, le facteur de correction de Cuningham [1] doit être inclus dans la loi de Stokes :

$$
K_{\mathrm{p}}=(1+A \lambda / a) Q_{\mathrm{p}} / 6 \pi \eta a
$$

ici $\lambda$ est le libre parcours moyen moléculaire et $\boldsymbol{A}$ une constante adimensionnelle. Pour l'air aux conditions normales, $A=0,86$ et $\lambda \sim 0,1 \mu \mathrm{m}$.

Il faut distinguer deux mécanismes de charge selon la taille des particules. Pour des diamètres supérieurs à $0,5 \mu \mathrm{m}$ on a charge par champ, et la charge limite est

$$
Q_{\mathrm{p}}=4 \pi \varepsilon_{0}\left[3 \varepsilon_{\mathrm{p}} /\left(\varepsilon_{\mathrm{p}}+2 \varepsilon_{0}\right)\right] E_{\mathrm{c}} a^{2}
$$

$E_{\mathrm{c}}$ étant le champ de charge et $\varepsilon_{\mathrm{p}}$ la permittivité des particules. Pour des diamètres inférieurs à $0,2 \mu \mathrm{m}$ la charge se fait par diffusion [1]. Dans l'intervalle, 0,2 à $0,5 \mu \mathrm{m}$, nous utilisons la loi semi-empirique de Cochet [14] :

$$
\begin{aligned}
Q_{\mathrm{p}} & =\left[\left(1+\lambda_{\mathrm{i}} / a\right)^{2}+\right. \\
& \left.+2\left(\varepsilon_{\mathrm{p}}-\varepsilon_{0}\right) /\left(1+\lambda_{\mathrm{i}} / a\right)\left(2 \varepsilon_{0}+\varepsilon_{\mathrm{p}}\right)\right] 4 \pi \varepsilon_{0} E_{0} a^{2}
\end{aligned}
$$

où $\lambda_{\mathrm{i}}$ est le libre parcours moyen des ions dans l'air $\left(\lambda_{\mathrm{i}} \sim 0,1 \mu \mathrm{m}\right)$. La variation de $M$ en fonction de la taille des particules est représentée sur la figure 3 pour différentes valeurs du champ de charge $E_{\mathrm{c}}$ (on a supposé que pour les particules $\varepsilon_{\mathrm{p}}=$ $2 \varepsilon_{0}$ et on s'est placé dans des conditions typiques de température : $T=130^{\circ} \mathrm{C}$ ). Pour les particules suffisamment petites la mobilité est très inférieure à la mobilité des ions et $M$ prend des valeurs élevées, du même ordre que celles pour les ions dans les liquides. L'importance des fortes valeurs du paramètre $M$ se reflète de deux façons différentes. Pour des particules suffisamment petites (de rayon $a<3 \mu \mathrm{m}$ pour fixer les idées) la mobilité est faible ce qui correspond à une valeur de la tension d'instabilité de 100 à 1000 fois plus faible que les tensions usuelles dans les PES. On sait que dans ces conditions une charge d'espace injectée produit une turbulence très intense dont l'échelle de vitesse est $w^{\prime} \sim$ $(1 / 3)(\varepsilon / \rho)^{1 / 2} \bar{E}$.

L'autre aspect du problème est relatif à l'entraînement des particules par l'écoulement turbulent. La 


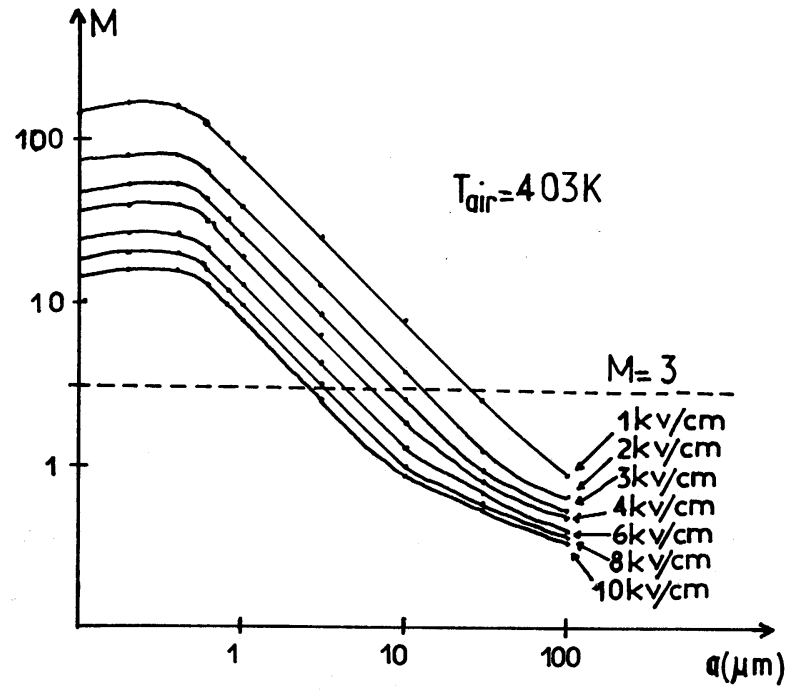

Fig. 3. - Variation du paramètre de mobilité $M=$ $(\varepsilon / \rho)^{1 / 2} / K_{\mathrm{p}}$ en fonction du rayon de la particule pour différents champs de charge $\left(T_{\text {air }}=403^{\circ} \mathrm{K}\right)$.

[Mobility parameter $M=(\varepsilon / \rho)^{1 / 2} / K_{\mathrm{p}}$ as a function of particle radius for different charging electric fields $\left(T_{\text {air }}=403^{\circ} \mathrm{K}\right)$.]

vitesse propre de ces particules chargées par rapport au liquide est $K \bar{E} \ll w^{\prime}\left(w^{\prime} / K \bar{E} \sim M\right)$. Elles peuvent donc être entraînées contre l'action du champ et subir une « diffusion » turbulente qui va bouleverser totalement les trajectoires régulières que l'on aurait avec un écoulement laminaire. Il faut donc s'attendre à des interactions très fortes entre écoulement et répartition des particules. Cette situation est tout à fait similaire à celle des ions dans un liquide, et une simulation du fonctionnement des PES avec des liquides apparait donc possible.

\section{Simulation expérimentale.}

L'utilisation d'un liquide ne permet pas une transposition tout à fait générale car dans un PES coexistent souvent des particules chargées et des ions. Nous allons donc considérer le cas particulier d'un PES à deux étages où les processus de charge et de collection sont séparés et pour lequel la simulation apparaît satisfaisante. La géométrie de la cellule utilisée est la suivante : un fil fin en tungstène

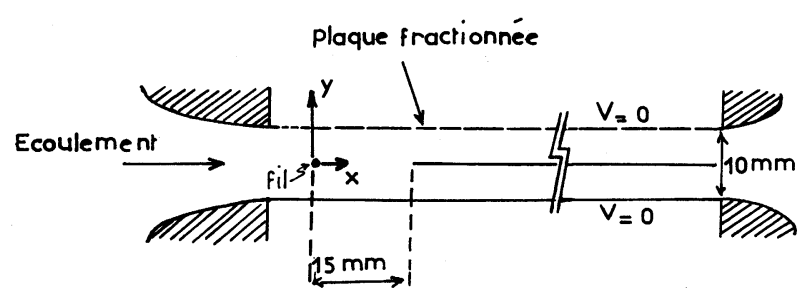

Fig. 4. - Schéma de la cellule d'étude.

[Schematic of the test cell.] $(\varnothing=0,1 \mathrm{~mm})$ et une plaque en cuivre d'épaisseur $1 \mathrm{~mm}\left(74 \times 400 \mathrm{~mm}^{2}\right)$ sont situés à égale distance entre deux électrodes en cuivre $\left(74 \times 500 \mathrm{~mm}^{2}\right)$ planes et parallèles (Fig. 4). Une des deux électrodes a été fractionnée en bandes minces électriquement isolées pour effectuer des mesures de densité de courant. Dans le liquide, les ions (qui jouent le rôle des particules chargées dans le gaz) sont injectés par le fil porté à haute tension $\left(V_{\mathrm{f}}\right)$. Le liquide utilisé est de l'huile silicone 47V10 (fourni par Rhône-Poulenc) dont les caractéristiques sont les suivantes :

$$
\nu=10^{-5} \mathrm{~m}^{2} / \mathrm{s} ; \rho=930 \mathrm{~kg} / \mathrm{m}^{3} ; \varepsilon_{\mathrm{r}}=2,6 .
$$

Près du fil vont se manifester les jets "chargés » fil-plaque, les jets pariétaux, et une turbulence intense. On admet qu'à une certaine distance dépendant de la vitesse moyenne $\bar{U}$ d'écoulement et de $V_{\mathrm{f}}$, on a un mélange suffisant et une distribution des ions analogue à celle des particules sortant de la zone de charge. Dans le second étage l'analogie est excellente puisque le liquide utilisé est isolant $\left(\rho \sim 10^{14} \Omega . \mathrm{cm}\right)$ et les seuls porteurs de charge mis en jeu sont les ions injectés par le fil.

Dans la pratique il y a quelques perturbations dues à la conduction résiduelle du liquide et à l'injection éventuelle par le plan et par le bord d'attaque de la plaque. Une correction est donc nécessaire pour obtenir la distribution de la densité de courant due seulement aux ions injectés par le fil. L'injection par le bord d'attaque où le champ électrique prend des valeurs importantes à cause de la forte courbure entraînait des perturbations notables dans le second étage. Nous sommes parvenus à éliminer complètement cet effet d'injection parasite en recouvrant soigneusement l'arête et son voisinage (sur $5 \mathrm{~mm}$ ) avec du film mince de téflon (d'épaisseur $\simeq 50 \mu \mathrm{m})$.

\section{Résultats expérimentaux.}

Les expériences ont été réalisées en faisant varier trois paramètres : la tension sur le fil $V_{\mathrm{f}}$, la vitesse moyenne d'écoulement $\bar{U}$, et la tension sur la plaque centrale $V_{\mathrm{p}}$ (c'est-à-dire le champ entre plaques). Sur la figure 5 , nous présentons une schématisation de la courbe de décroissance de la densité de courant due aux ions entrant dans la zone de collection. On distingue trois parties : une première zone s'étendant jusqu'à $x_{1}\left(10 d<x_{1}<30 d\right)$ où la densité de courant passe par un maximum puis décroît rapidement. Le maximum correspond à " l'impact » du jet chargé ; c'est dans ce jet qu'est créé l'essentiel de la turbulence. La zone de décroissance est associée au développement du jet pariétal. Les phénomènes dans cette première zone sont similaires à ceux déjà observés en géométrie fil entre deux plaques [12]. Dans la deuxième zone la densité de courant décroît exponentiellement : $j(x) \alpha \exp (-x / L)$. Comme la 


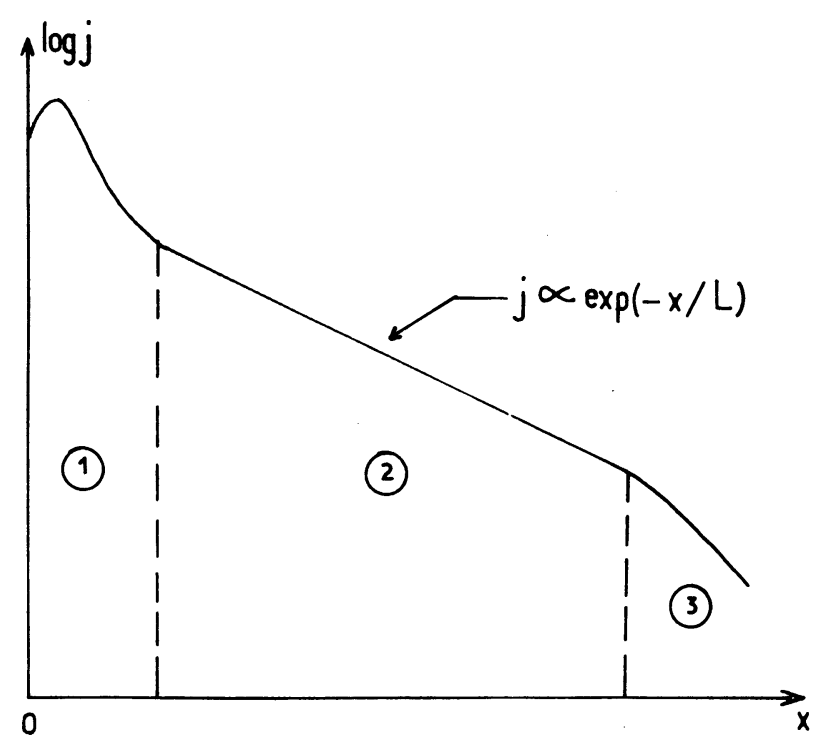

Fig. 5. - Schématisation de la courbe de décroissance de la densité de courant due à la décharge des ions entrant dans la zone de collection.

[Schematic of the decreasing current density due to discharge of ions entering the collection zone.]

majeure partie de la charge injectée a déjà été collectée, on peut supposer que la charge d'espace qui subsiste est peu importante et donc admettre que le champ est uniforme et que la vitesse $w_{\mathrm{E}}=$ $K \bar{E}$ est elle aussi constante. Dans la troisième zone la densité de courant se remet à décroître plus rapidement. La caractérisation est cependant malaisée car les corrections deviennent rapidement du même ordre de grandeur que le courant mesuré. C'est la deuxième zone à décroissance exponentielle qui est la plus intéressante ; elle va nous permettre de comparer les résultats expérimentaux aux diverses prédictions théoriques.

Dans la théorie de Léonard et al., à distance suffisamment grande on a une variation du rendement de type exponentiel (Fig. 1) ; cette variation correspond à la décroissance exponentielle en $\exp (-x / L)$ des quantités de particules collectées. Cependant la longueur caractéristique $L$ est fonction du nombre de Peclet comme le montre la figure 6 (les valeurs de $L$ sont déduites des courbes de la figure 1). On peut donc écrire :

$$
L=(\bar{U} d / K \bar{E}) f(P e)
$$

avec $P e=K \bar{E} d / D_{\mathrm{t}}=K V_{\mathrm{p}} / D_{\mathrm{t}}$, où $V_{\mathrm{p}}$ est la tension sur la plaque centrale.

Nos conditions expérimentales n'ont pas permis de faire varier la vitesse moyenne $\bar{U}$ de l'écoulement dans de grandes proportions. Nous avons cependant vérifié que la longueur caractéristique de décroissance de $j$ est proportionnelle à $\bar{U}$ comme le prévoit

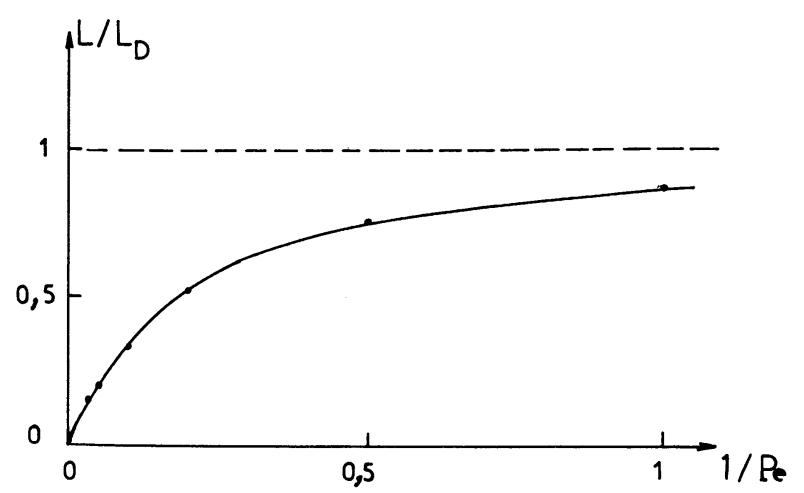

Fig. 6. - Variation de la longueur caractéristique $L$ (déduite des courbes de la figure 1) en fonction de l'invese du nombre de Peclet.

[Characteristic length $L$ (deduced from curves of Fig. 1) as a function of the inverse of the Peclet number.]

(1). Pour mettre en évidence l'effet du champ de collection sur la décroissance de $j(x)$ et sur $L$, nous avons effectué des séries de mesures en fixant $\bar{U}$ et $V_{\mathrm{f}}$ et en faisant varier $V_{\mathrm{p}}$. Nous présentons sur la figure 7 un réseau de courbes pour $\bar{U}=19,2 \mathrm{~cm} / \mathrm{s}$ et $V_{\mathrm{f}}=10 \mathrm{kV}$. Lorsqu'on augmente le champ, la

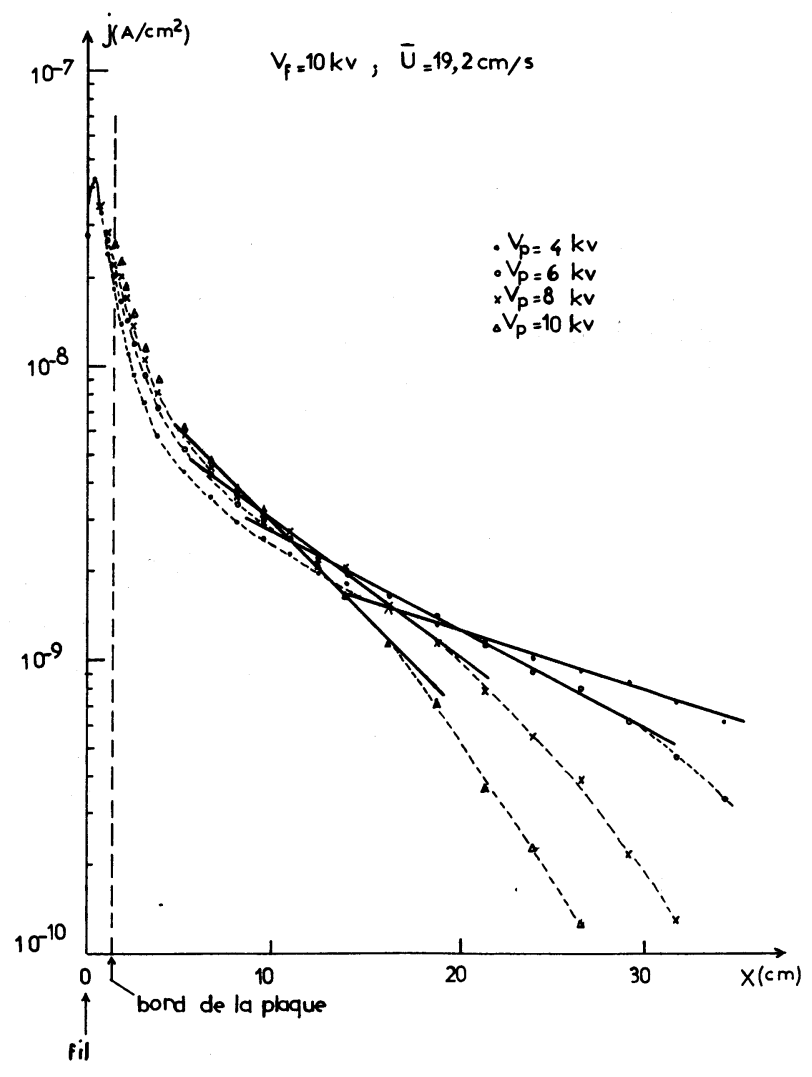

Fig. 7. - Distribution de la densité de courant pour différents champs de collection: pour $\bar{U}=19,2 \mathrm{~cm} / \mathrm{s}$, $V_{\mathrm{f}}=10 \mathrm{kV}$.

[Distribution of current density for different collection fields : $\bar{U}=19.2 \mathrm{~cm} / \mathrm{s}, V_{\mathrm{f}}=10 \mathrm{kV}$.] 


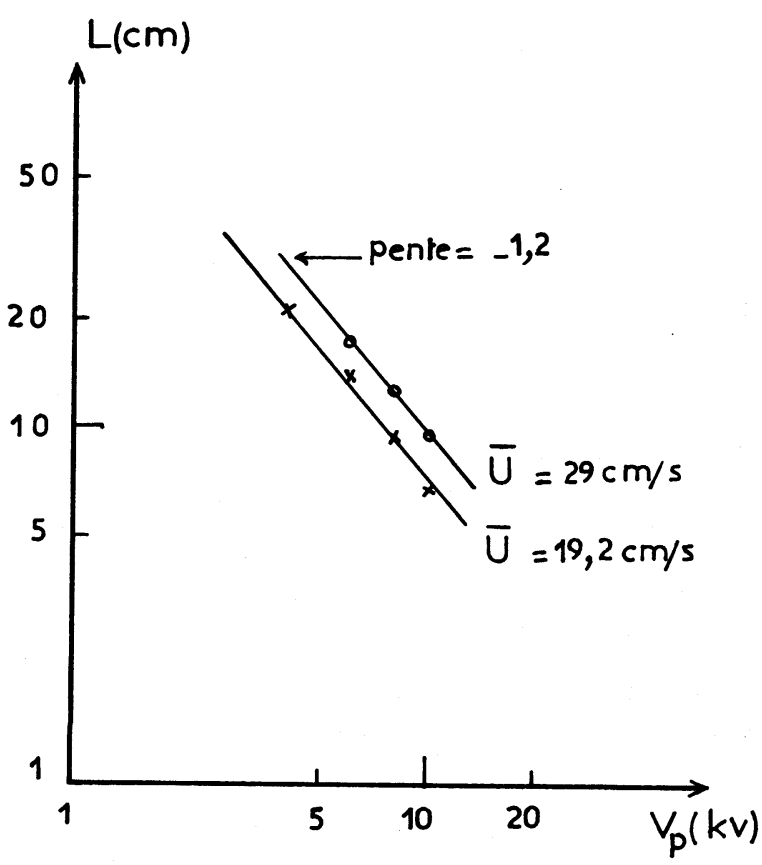

(a) $V_{f}=10 \mathrm{kv}$

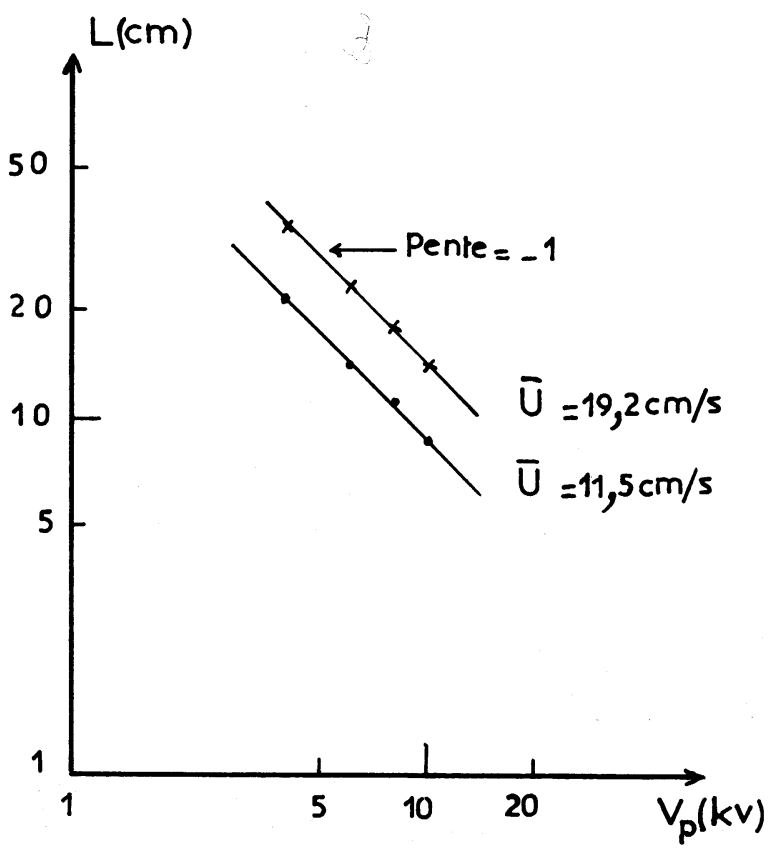

(b) $V_{f}=20 \mathrm{kv}$

Fig. 8. - Longueur caractéristique $L$ en fonction de la tension $V_{\mathrm{p}}$ sur la plaque centrale. a) $V_{\mathrm{f}}=10 \mathrm{kV}$; $\bar{U}=19,2$ et $29 \mathrm{~cm} / \mathrm{s}$. b) $V_{\mathrm{f}}=20 \mathrm{kV} ; \bar{U}=11,5$ et $19,2 \mathrm{~cm} / \mathrm{s}$.

[Characteristic length $L$ as a function of the applied voltage $V_{\mathrm{p}}$ on the central plate electrode. a) $V_{\mathrm{f}}=$ $10 \mathrm{kV} ; \bar{U}=19.2$ and $29 \mathrm{~cm} / \mathrm{s}$. b) $V_{\mathrm{f}}=20 \mathrm{kV} ; \bar{U}=11.5$ and $19.2 \mathrm{~cm} / \mathrm{s}$.]

vitesse de migration des porteurs de charge augmente ; on constate que dans la première zone (ici pour $x<x_{1} \approx 16 d$ ) la densité de courant augmente avec $V_{\mathrm{p}}$. Suffisamment loin, par contre, $(x>30 d$ à $40 d)$ c'est le contraire ; ceci est dû au fait que dans la zone de décroissance exponentielle la longueur caractéristique $L$ diminue quand $V_{\mathrm{p}}$ croît. En portant $L$ en fonction de $V_{\mathrm{p}}$ (Fig. 8) on observe que $L$ varie comme $V_{\mathrm{p}}^{-\alpha}$ avec $\alpha=1$ pour $V_{\mathrm{f}}=$ $20 \mathrm{kV}$ (Fig. 8b) et $\alpha=1,2$ pour $V_{\mathrm{f}}=10 \mathrm{kV}$ (Fig. 8a). Nous allons voir que cette différence est significative et provient du fait que $L$ varie comme $V_{\mathrm{p}}^{-1} f\left(K V_{\mathrm{p}} / D_{\mathrm{t}}\right), f$ étant une fonction décroissante de $P e \propto V_{\mathrm{p}}$ (voir Fig. 6).

On peut isoler l'influence du nombre de Peclet en travaillant à vitesse et champ de collection fixés et en faisant varier la tension sur le fil injecteur. L'injection et l'intensité turbulente correspondante et par conséquent la diffusivité turbulente $D_{\mathrm{t}}$ vont varier avec $V_{\mathrm{f}}$. Le réseau de courbes de la figure 9 montre que $j(x)$ augmente avec $V_{\mathrm{f}}$ pour toutes valeurs de $x$. De plus la longueur caractéristique de la zone à décroissance exponentielle augmente avec $V_{\mathrm{f}}$. La figure 10 montre que $L$ varie d'abord sensiblement linéairement avec $V_{\mathrm{f}}$ puis tend à se saturer. Cette variation de $L$ est en accord qualitatif avec la prédiction déduite de la théorie de Léonard et al. (Fig. 6). Ce comportement permet de comprendre la différence observée précédemment sur la valeur de l'exposant $\alpha$ : pour $V_{\mathrm{f}}=20 \mathrm{kV}$ la fonction $f(\mathrm{Pe})$ devient indépendante de $V_{\mathrm{p}}$, ce qui entraîne $L=$ $\mathrm{Cte} / V_{\mathrm{p}}$ tandis que pour $V_{\mathrm{f}}=10 \mathrm{kV}, f(P e)$ varie avec $V_{\mathrm{p}}$. Si on admet que la valeur de saturation de la figure 10 est la longueur de Deutsch, on trouve expérimentalement $L_{\mathrm{D}} \approx 20 \mathrm{~cm}$. Cette valeur est tout à fait comparable à l'estimation $16 \mathrm{~cm}<L_{\mathrm{D}}<$ $24 \mathrm{~cm}$ faite avec l'estimation de la mobilité ionique $2 \times 10^{-9} \mathrm{~m}^{2} / \mathrm{v} . \mathrm{s}<K<3 \times 10^{-9} \mathrm{~m}^{2} / \mathrm{v} . \mathrm{s}$.

\section{Conclusion.}

La théorie de Léonard et al. [2] semble en première approximation décrire correctement les phénomènes dans la zone de collection du dispositif expérimental. Il est nécessaire cependant de compléter l'étude actuelle, d'abord en déterminant avec une meilleure précision la mobilité vraie des ions dans l'huile silicone $47 \mathrm{~V} 10$ et ensuite en étudiant l'écoulement par anémométrie laser-Doppler pour mesurer les vitesses fluctuantes et estimer la diffusivité turbulente $D_{\mathrm{t}}$. Ceci permettra de faire une comparaison quantitative et non plus seulement qualitative du rôle du nombre de Peclet.

La simulation EHD utilisée ici est beaucoup moins satisfaisante dans le cas des précipitateurs à un seul étage où une nappe de fils permet à la fois 


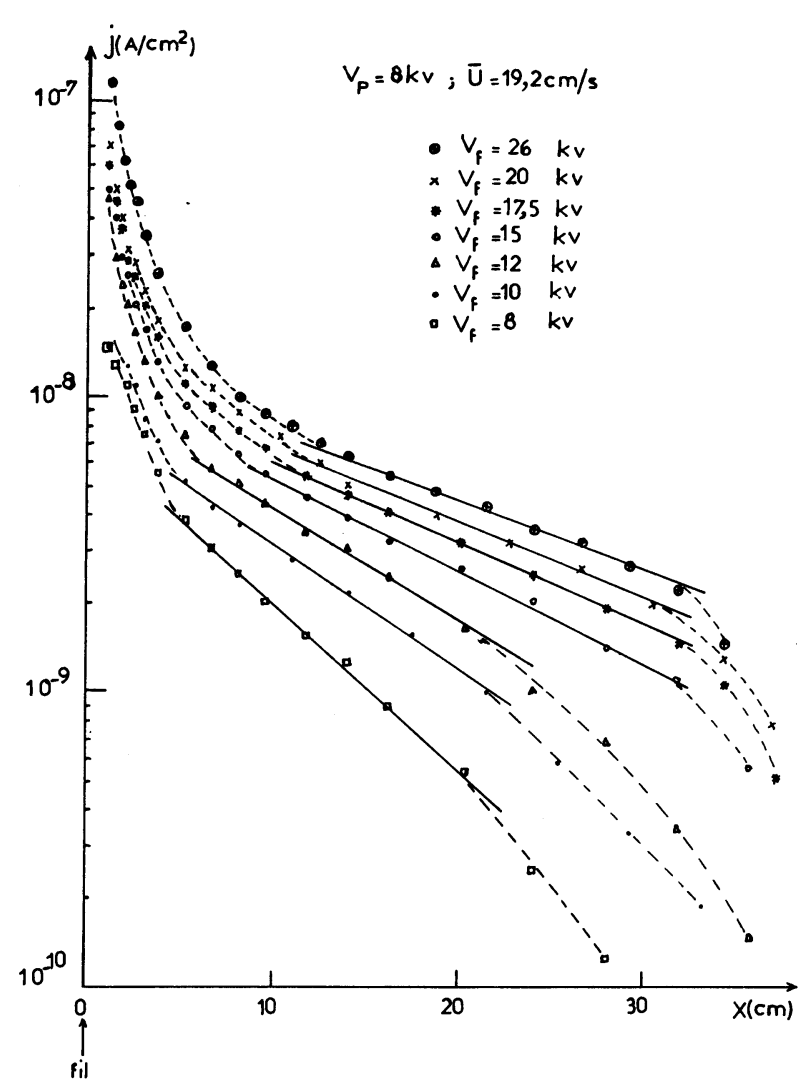

Fig. 9. - Distribution de la densité de courant pour différentes tensions sur le fil injecteur $V_{\mathrm{f}}: \bar{U}=19,2 \mathrm{~cm} / \mathrm{s}$, $V_{\mathrm{p}}=8 \mathrm{kV}$.

[Distribution of current density for different applied voltages on the injecting wire $V_{\mathrm{f}}: \bar{U}=19.2 \mathrm{~cm} / \mathrm{s}, V_{\mathrm{p}}=$ $8 \mathrm{kV}$.

d'imposer le champ électrique précipitant et de créer, par effet couronne, les ions qui chargent les particules. Pour ces précipitateurs les ions injectés de façon périodique dans l'espace vont induire de gros rouleaux de convection $[4,5]$. Il ne fait pas de doute que la turbulence ainsi engendrée est vigoureuse et que le nombre de Peclet prend des valeurs faibles de sorte que la théorie de Deutsch doit s'appliquer en première approximation (voir également [3]).

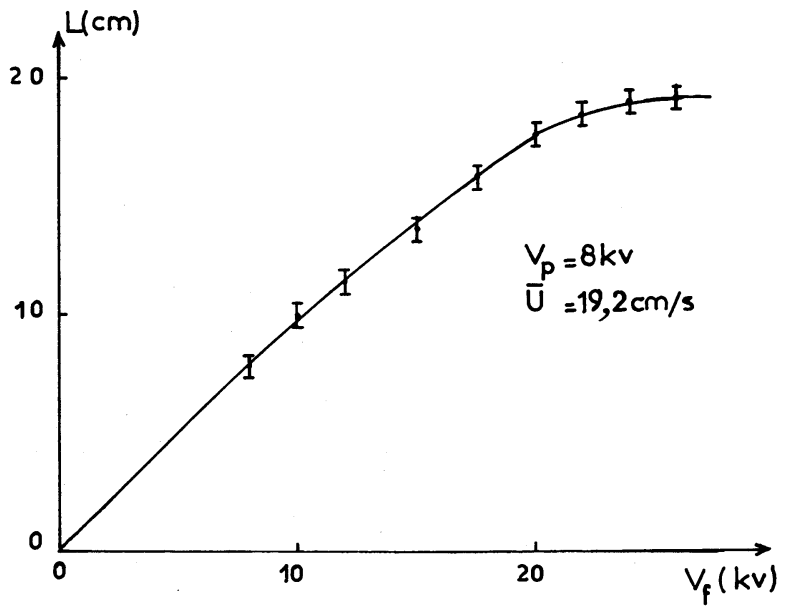

Fig. 10. - Variation de la longueur caractéristique $L$ en fonction de la tension sur le fil injecteur $V_{\mathrm{f}}: \bar{U}=$ $19,2 \mathrm{~cm} / \mathrm{s}$ et $V_{\mathrm{p}}=8 \mathrm{kV}$.

[Characteristic length $L$ as a function of the applied voltage on the injecting wire $V_{f}: \bar{U}=19.2 \mathrm{~cm} / \mathrm{s}, V_{\mathrm{p}}=$ $8 \mathrm{kV}$.]

Il apparaît donc que la prise en compte de la turbulence ne permet pas d'expliquer que très souvent dans les applications pratiques on trouve une longueur effective $L$ nettement supérieure à $L_{\mathrm{D}}$. Si on ne met pas en doute cette affirmation un tel effet peut provenir par exemple d'un processus de réentraînement de particules déjà collectées. Il nous semble cependant que cette observation $\left(L>L_{\mathrm{D}}\right)$ devrait être réexaminée soigneusement. En effet la plupart des auteurs comparent la vitesse effective $w_{\mathrm{E}}$ des particules chargées telle qu'on la déduit du rendement du précipitateur à la valeur théorique obtenue en supposant que les particules ont acquis la charge limite (charge par champ). Cette hypothèse est peut-être très optimiste car les particules se déplacent dans un domaine où le champ électrique n'est pas uniforme. Dans ces conditions, même avec des particules toutes identiques, il est vraisemblable que l'on a une distribution assez étendue pour la charge qu'elles portent.

\section{Bibliographie}

[1] White, H. J., Industrial Electrostatic Precipitation (Addison-Wesley, Reading, Ma), 1963.

[2] Leonard, G. L., Mitchner, M. et Self, S. A., Particle Transport in Electrostatic Precipitators, Atmos. Environ. 14 (1980) 1289-1299.

[3] Self, S. A., Mitchner, M., Khim, K. D., Choi, D. H. et LEACH, R., Effect of Turbulence on Precipitator Performance, Presented at Second
International Conference on Electrostatic Precipitation, Kyoto, Japan, Nov. 1984.

[4] Yamamoto, T. et VelkofF, H. R., Electrohydrodynamics in an Electrostatic Precipitator, J. Fluid Mech. 108 (1981) 1-18.

[5] Leonard, G. L., Mitchner, M. et Self, S. A., Experimental Study of the Electrohydrodynamic Flow in Electrostatic Precipitators, J. Fluid Mech. 127 (1983) 123-140. 
[6] Atten, P., Lahjomri, A. C. et MacCluskey, F., The Electrohydrodynamic Origin of Turbulence in Electrostatic Precipitators, à paraître dans IEEE Trans. Industrial Appl., 1986.

[7] FARADAy, M., Experimental Researches, Dover, 1, 1838.

[8] Atten, P., MoreaU, R., Stabilité électrohydrodynamique des liquides isolants soumis à une injection unipolaire, J. Méc. 11 (1972) 471-520.

[9] SCHNEIDER, J. M., WATSON, P. K., Electrohydrodynamic Stability of Space Charge Limited Currents in Dielectric Liquids : 1-Theoretical Study, Phys. Fluids 13 (1970) 1948-1954.

[10] Lacroix, J. C., Atten, P. et Hopfinger, E. J., Electroconvection in a dielectric liquid layer subjected to unipolar injection, J. Fluid Mech. 69 (1975) 539-563.
[11] Atten, P. et Haïdara, M., Electrical Conduction and EHD Motion of Dielectric Liquids in a Blade-plane Electrodes Assembly, IEEE Trans. Electr. Insul. EI-20 (1985) 187-198.

[12] MCCluskey, F. M. J. et AtTen, P., Entrainment of an Injected Unipolar Space Charge by a Forced Flow in a Rectangular Channel, J. Electrostatics 15 (1984) 329-342.

[13] McCluskey, F. M. J. et Atten, P., Velocity Profiles in the Injection Zone of an E.H.D. Generator and Efficiency Considerations, IEEE Trans. Electr. Insul. EI-20 (1985) 405-412.

[14] Cochet, R., Lois de charge des fines particules (submicroniques), La Physique des forces électrostatiques et leurs applications (Ed. C.N.R.S., Paris) 1961, pp. 331-338. 